\title{
Tratamiento de la enfermedad de Kienböck juvenil mediante fijación temporal escafo-trapezoidea
}

\author{
A. Guijarro Valtueña, A. García Olea, A. Hualde \\ Servicio de Cirugía Ortopédica y Traumatología. Hospital Universitario Puerta de Hierro Majadahonda. Madrid. España.
}

\begin{abstract}
Resumen: No existe un consenso acerca del tratamiento de la enfermedad de Kienböck en el adolescente. Se presenta un caso de un paciente de 14 años que fue tratado de manera satisfactoria con una fijación temporal entre trapezoide y escafoides usando tres agujas de Kirschner que se mantuvieron 6 meses. El seguimiento radiológico mostró recuperación de la intensidad de señal en resonancias magnéticas seriadas. A los dos años de la cirugía el paciente no tiene dolor y ha recuperado un rango articular completo
\end{abstract}

Palabras claves: Kienböck juvenil, artrodesis temporal.

\section{Juvenile lunatomalacia treated by temporary scaphotrapezoidal joint fixation}

\begin{abstract}
There is no consensus about the correct treatment of the adolescent Kienböck's disease. We report a case of a 14-year-old boy who was treated successfully by temporary scaphotrapezoidal fixation using three K-wires during six months. Radiologic follow-up shows signal intensity recovery in successive MRI images. At two years after surgery the patient does not complain of wrist pain or stiffness.
\end{abstract}

Key words: Juvenile lunatomalacia, temporal fixation.

La enfermedad de Kienböck según fue descrita inicialmente en 1910, es una lunatomalacia que se presenta en pacientes que en general son varones de entre 20 a 40 años. Aunque la etiología parece estar en relación con una necrosis avascular, aún se desconoce la causa inicial', y se han formulado numerosas teorías, que van desde problemas circulatorios primarios o traumatismos hasta un aumento de la presión intraósea. Se han propuesto numerosas formas de tratamiento basadas en el conocimiento de la fisiopatología de la enfermedad para intentar frenar su avance o para paliar sus secuelas.

La sintomatología típica es el dolor en la región del semilunar dorsal y la rigidez de la muñeca. El diagnóstico se hace mediante pruebas complementarias; con la radiología simple pueden observar cambios progresivos que sirven para clasificar la enfermedad en los cuatro estadios descritos por Lichtman². Sin embargo, la Resonancia Magnética (RMN) es la técnica más sensible y específica para el diagnóstico de osteonecrosis ${ }^{3}$ puesto que la radiología simple puede ser normal en estadios iniciales.

No existe una evidencia clara que sustente una única forma de tratamiento, muchos pacientes mejoran con inmovilización temporal pero esto no detiene la pro- gresión de la enfermedad. Otras técnicas incluyen escisión del semilunar, injertos vascularizados, osteotomías de realineación del radio ${ }^{4} y$ artrodesis parciales o totales de muñeca.

Se han publicado algunas pequeñas series de casos de esta enfermedad en niños ${ }^{5}$. El tratamiento conservador en niños menores de 12 años ha dado buenos resultados; sin embargo, en los mayores de 12 años, se hizo necesario el tratamiento quirúrgico en un segundo tiempo ${ }^{6}$. Las técnicas quirúrgicas son similares a las descritas en adultos aunque hay algunos autores que han intentado técnicas temporales menos agresivas $^{7}$ entre las que destacan las fijaciones transitorias entre los huesos del carpo en un intento de descomprimir el semilunar.

Se presenta un caso de enfermedad de Kienböck en un paciente en edad juvenil que fue tratado con una técnica de artrodesis temporal para mantener la altura
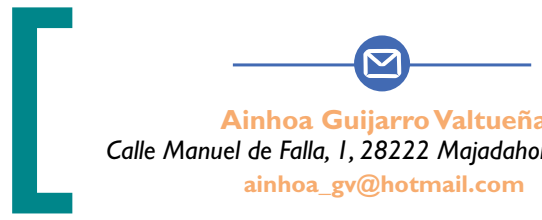

Calle Manuel de Falla, I, 28222 Majadahonda, Madrid ainhoa_gv@hotmail.com 

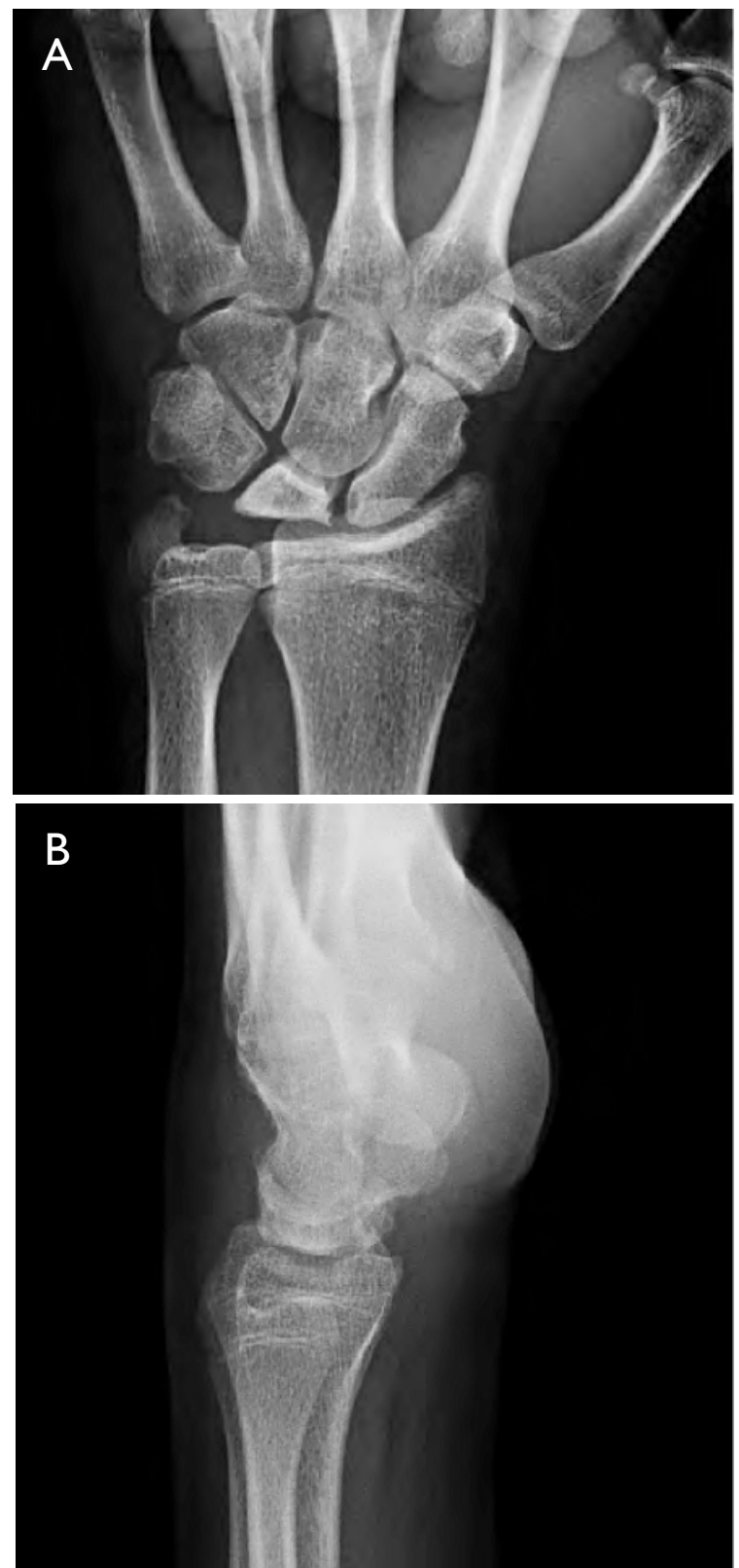

Figura I. Radiología simple al comienzo de los síntomas del paciente. A. Radiografía anteroposterior en la que se observa esclerosis del semilunar y pérdida de altura $(0,46 \mathrm{~cm})$. B. Radiografía lateral en la que se observa ángulo radioescafoideo de $54^{\circ}$.

del carpo mientras se da tiempo a la revascularización del semilunar.

\section{CASO CLÍNICO}

Se presenta el caso de un varón de 14 años que consultó por dolor en la muñeca izquierda de diez
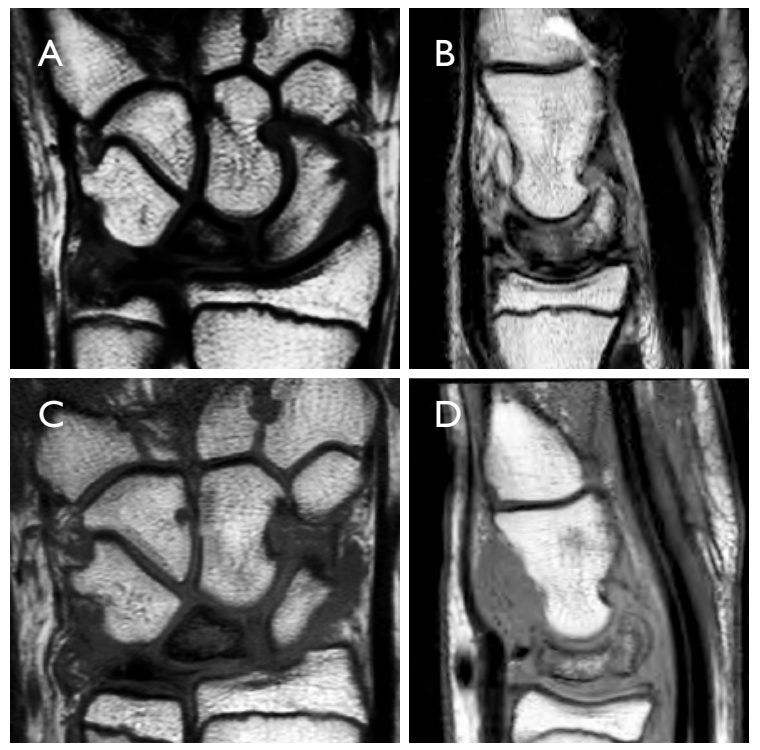

Figura 2. Estudio mediante Resonancia Magnética Nuclear. A y B. Estudio al comienzo de los síntomas. C y D. Estudio al terminar el tratamiento conservador.

meses de evolución que se agravó tras un esfuerzo practicando deporte. El paciente aporta una radiografía realizada al inicio de los síntomas informada como normal.

La exploración física reveló dolor en la parte dorsal del carpo, un rango articular limitado a $30^{\circ}$ de flexión palmar y $60^{\circ}$ de flexión dorsal. Se midió la fuerza de presa con el dinamómetro de Jamar® en $10 \mathrm{~kg}$ en la mano izquierda y $42 \mathrm{~kg}$ en la mano derecha. El Quick DASH tuvo un resultado de 86 puntos.

Fue realizada una radiografía simple en la que se podía apreciar esclerosis y colapso del semilunar (2) Figura I. Se realizaron varias mediciones: la altura del semilunar fue de $0,46 \mathrm{~cm}$, el ángulo radioescafoideo fue de $54^{\circ}$ y el índice de Youm se calculó en 0,48.

Por todos estos hallazgos se clasificó la enfermedad en estadio IIIA según la clasificación de Lichtman.

Se inició un tratamiento conservador con yeso cerrado durante seis semanas seguido de una ortesis a tiempo parcial otros tres meses. Transcurrido este tiempo, el dolor y la rigidez no habían mejorado y la RMN mostraba persistencia del edema óseo (a) Figura 2.

Dado el fracaso del tratamiento conservador, se planteó tratamiento quirúrgico. En un intento por no perturbar el crecimiento óseo y evitar artrotomías y artrodesis, se propuso una fijación temporal escafo- 

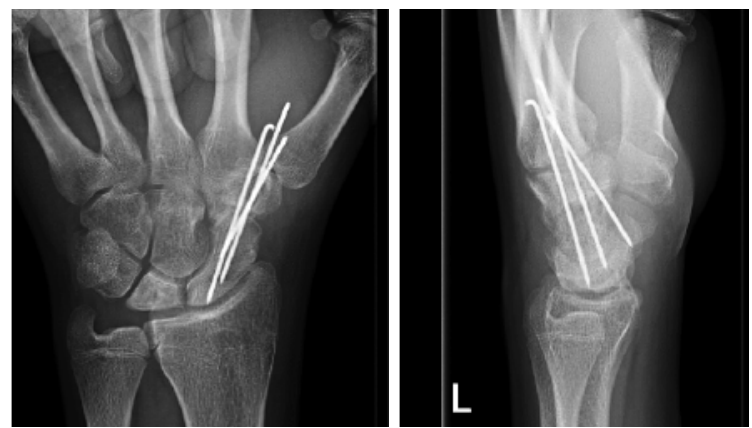

Figura. 3. Fijación temporal escafo-trapezoidal con agujas de Kirschner de 1,5 mm.

trapezoidal con agujas de Kirschner de 1,5 mm de diámetro de manera percutánea para mantener la altura del hueso semilunar (- Figura 3. El paciente fue inmovilizado tres semanas más.

A los 3 meses el dolor y el edema óseo habían mejorado. A los 6 meses de la cirugía se retiraron las agujas de Kirschner por intolerancia en los puntos de entrada. La radiografía simple y la resonancia magnética mostraron signos de buena recuperación con disminución de la zona de necrosis (a) Figura 4.

Al año de la cirugía, la longitud del semilunar mejoró hasta $0,74 \mathrm{~cm}$ y el índice de Youm volvió a calcularse en 0,51 (2) Figura 5.

Transcurridos dos años de la cirugía, el paciente está haciendo su vida habitual sin dolor, salvo actividad deportiva de contacto. La fuerza de presa de puño medida con el dinamómetro de Jamar ${ }^{\circledR}$ en tres mediciones seguidas fue de 46,44 y $36 \mathrm{Kg}$ en la mano izquierda. El cuestionario Quick DASH mejoró desde 86 puntos antes de la cirugía hasta 27 puntos después de dos años. En el estudio radiológico se observa una recuperación prácticamente completa de la necrosis del semilunar (- Figura 6 .

\section{DISCUSIÓN}

La enfermedad de Kienböck es un proceso degenerativo que cursa con dolor y disfunción de la articulación de la muñeca. Todavía no se conoce con claridad la etiología aunque se han propuesto muchos factores que contribuyen a la necrosis avascular, como un traumatismo, una fractura, una lesión ligamentosa o un trastorno de la circulación primario'. Lo que parece más claro es que cursa con un aumento de la presión intraósea, aunque no está claro si este incremento de la presión contribuye a la enfermedad o es secundario a la enfermedad misma ${ }^{8}$.

Los estudios de imagen son básicos para el diagnóstico de la enfermedad. La radiografía simple se usa para medir la varianza cubital, la inclinación radial, la altura del carpo, el ángulo radioescafoideo, el tamaño del semilunar y su morfología ${ }^{8}$. Al mismo tiempo sirve para el estadiaje de la enfermedad según Lichtman; que además tiene valor para la decisión del tratamiento. La resonancia magnética es útil en los estadios iniciales cuando la radiología simple es normal. Podemos apreciar disminución de la señal en secuencias TI y cambios en la señal en secuencias T2 dependiendo de la extensión de la enfermedad ${ }^{3}$.

En el presente caso se apreció en la radiografía simple un semilunar escleroso de $0,46 \mathrm{~cm}$ de tamaño, una altura del carpo estimada con el índice de Youm en 0,48 y un ángulo radioescafoideo de $54^{\circ}$. En la resonancia magnética se observó un cambio de señal
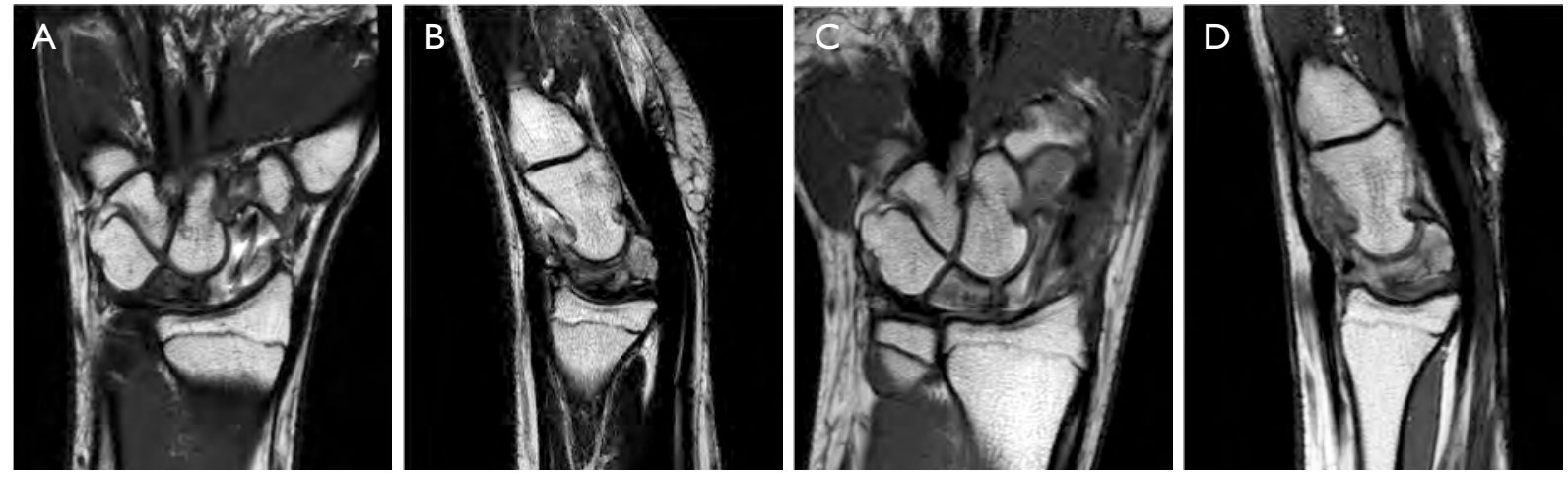

Figura 4. Estudio mediante RMN, se observa una disminución del edema óseo y zona de necrosis en el semilunar. A y B. I5 semanas tras la intervención. C y D. 24 semanas tras la intervención. 

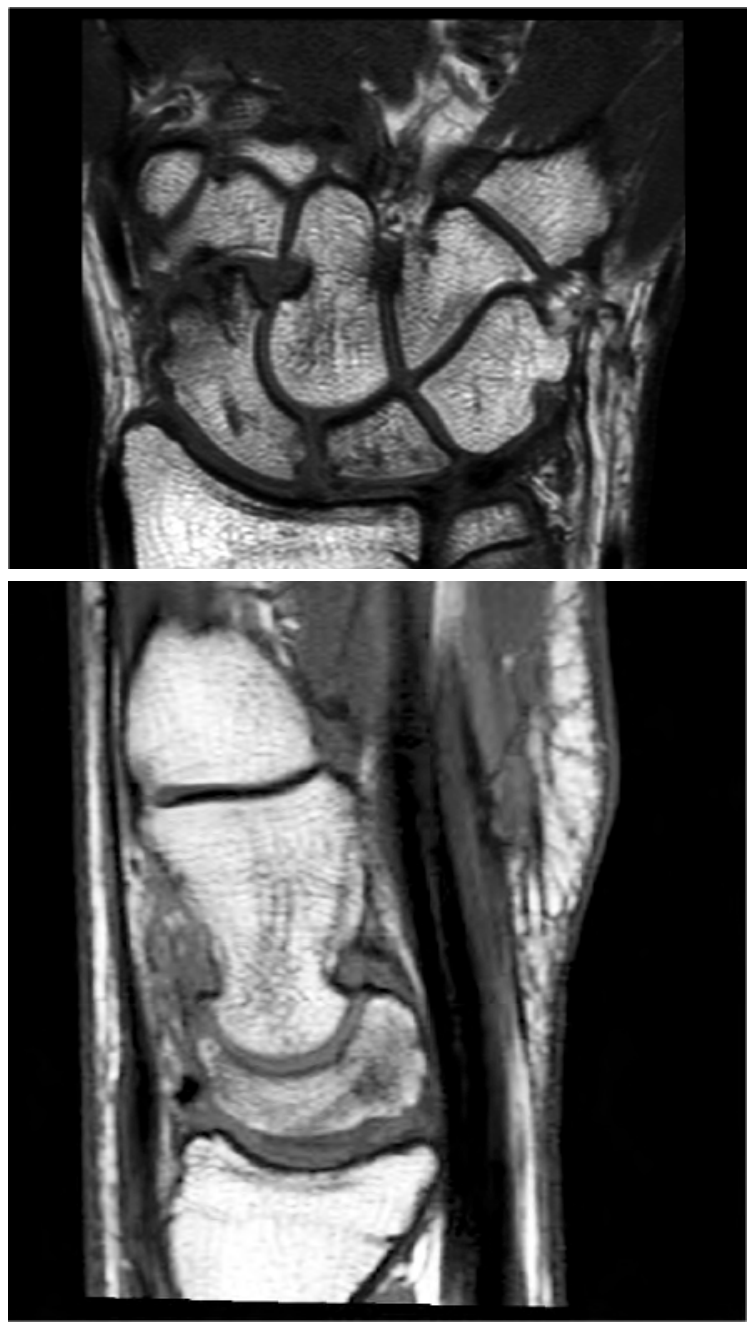

Figura 5. Estudio mediante RMN al año de la intervención quirúrgica. Se observan signos de buena recuperación con disminución de la zona de necrosis y normalización de la señal ósea.

en el semilunar que indica las áreas de necrosis. Por tanto, se clasificó al enfermo como estadio IIIA según Lichtman (colapso del semilunar con conservación de la altura y la alineación del carpo).

Aunque existen muchos estudios acerca de la progresión de la enfermedad ${ }^{\prime 2}$, no se conoce la historia natural de la enfermedad con precisión. Existen guías para el tratamiento según el estadio de la enfermedad, aunque la necesidad del tratamiento debe hacerse siempre en función de la sintomatología del paciente.

El tratamiento para la enfermedad de Kienböck en los adolescentes ha sido publicado en pequeñas series de casos. Ferlic y cols ${ }^{5}$ e Irisarri y cols. ${ }^{6}$ demostraron buenos resultados con inmovilización prolongada como
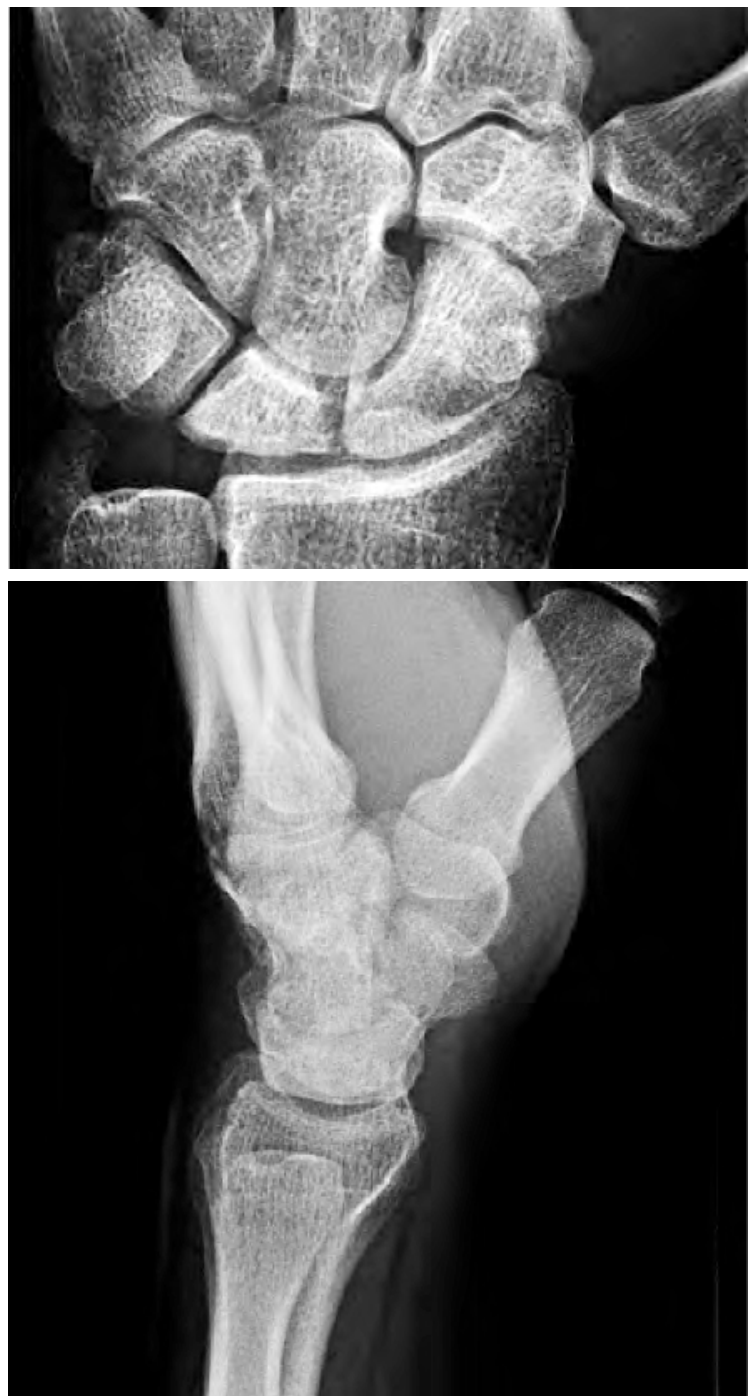

Figura 6. Radiografías simples a los dos años de la intervención quirúrgica.

se hizo con el caso del paciente presentado. Herzberg y cols ${ }^{9}$ publicaron el caso de una gimnasta de 14 años tratada con inmovilización durante 3 meses y posteriormente inmovilizada solo por la noche y en momentos de dolor con recuperación completa de la vascularización del semilunar. Sin embargo, la persistencia del dolor y la rigidez tras mantener al paciente inmovilizado durante un periodo largo de tiempo hizo que se propusiera tratamiento quirúrgico en este caso.

La osteotomía de acortamiento radial es la técnica quirúrgica que se usa más frecuentemente en los niños ${ }^{5}$. Ha demostrado disminuir la presión del semilunar en pacientes con varianza cubital negativa ${ }^{4}$, sin embargo, en pacientes con varianza neutra o positiva, este método aumenta el riesgo de impactación cubital. 
Ando y coll ${ }^{7}$ publicaron un estudio de seis pacientes con una edad media de 14 años en los que obtuvieron buenos resultados con una fijación temporal entre escafoides y trapezoide durante tres a seis meses con dos o tres agujas de Kirschner, encaminada a descomprimir el semilunar transfiriendo las cargas a través de la articulación hueso grande-escafoides-radio para darle el tiempo necesario al semilunar para recuperar la vascularización ${ }^{10}$.

Nosotros realizamos el mismo tratamiento, efectuamos la artrodesis con tres agujas de Kirschner y las mantuvimos seis meses con buenos resultados. Transcurridos dos años el paciente se encuentra libre de dolor y la enfermedad no ha progresado radiológicamente.

\section{CONFLICTOS DE INTERESES}

Los autores declaran no tener conflictos de intereses.

\section{BIBLIOGRAFÍA}

I. Williams CS, Gelberman RH.Vascularity of the lunate. Anatomic studies and implications for the development of osteonecrosis. Hand Clin. |993;9:39|-8.
2. Lichtman DM, Degnan GG. Staging and its use in the determination of treatment modalities for Kienböck's disease. Hand Clin. 1993;9:409- 16.

3. Schuind F, Eslami S, Ledoux P. Kienbock's disease. J Bone Joint Surg Br. 2008;90: $133-9$.

4. Afshar A, Eivaziatashbeik K. Long-term clinical and radiological outcomes of radial shortening osteotomy and vascularized bonegraft in Kienböck disease. J Hand Surg Am. 20 I 3;38:289-96.

5. Ferlic RJ, Lee DH, Lopez-Ben RR. Pediatric Kienböck's disease: case report and review of the literature. Clin Orthop Relat Res. 2003:408:237-44.

6. Irisarri C, Kalb K, Ribak S. Infantile and juvenile lunatomalacia. J Hand Surg EurVol. 20 I0;35:544-8.

7. Ando Y, Yasuda M, Kazuki K, Hidaka N, Yoshinaka Y. Temporary scaphotrapezoidal joint fixation for adolescent Kienböck's disease. J Hand Surg Am. 2009;34:14-9.

8. Lutsky K, Beredjiklian PK. Kienböck disease. J Hand Surg Am. 20 12;37:1942-52.

9. Herzberg GI, Mercier S, Charbonnier JP, Got P. Kienböck's disease in a 14-year-old gymnast: a case report.J Hand Surg Am. 2006;31:264-8.

10. Kazuki K, Uemura T, Okada M, Egi T.Time course of magnetic resonance images in an adolescent patient with Kienböck's disease treated by temporary scaphotrapezoidal joint fixation: a case report. J Hand Surg Am. 2006;31:63-77. 